\title{
Charmonium dynamics in heavy ion collisions
}

\author{
O. Linnyk ${ }^{1}$, E. L. Bratkovskaya ${ }^{1}$, W. Cassing ${ }^{2}$, H. Stöcker ${ }^{1,3}$ \\ ${ }^{1}$ Frankfurt Institute for Advanced Studies, Ruth-Moufang-Str. 1, 60438 Frankfurt \\ am Main, Germany \\ ${ }^{2}$ Institut für Theoretische Physik, Universität Giessen, Heinrich-Buff-Ring 16, 35392 \\ Giessen, Germany \\ ${ }^{3}$ Institut für Theoretische Physik, Johann Wolfgang Goethe University, \\ Max-von-Laue-Str. 1, 60438 Frankfurt am Main, Germany \\ E-mail: linnyk@fias.uni-frankfurt.de
}

\begin{abstract}
Applying the HSD transport approach to charmonium dynamics within the 'hadronic comover model' and the 'QGP melting scenario', we show that the suppression pattern seen at RHIC cannot be explained by the interaction with baryons, comoving mesons and/or by color screening mechanism. The interaction with hadrons in the late stages of the collision (when the energy density falls below the critical) gives a sizable contribution to the suppression. On the other hand, it does not account for the observed additional charmonium dissociation and its dependence on rapidity. Together with the failure of the hadron-string models to reproduce high $v_{2}$ of open charm mesons, this suggests strong pre-hadronic interaction of $c \bar{c}$ with the medium at high energy densities.
\end{abstract}




\section{1. $J / \Psi$ production vs suppression in different theoretical scenarios}

The microscopic Hadron-String-Dynamics (HSD) transport calculations (employed here) provide the correct space-time geometry of the nucleus-nucleus reaction and a rather reliable estimate for the local energy densities achieved. The energy density $\varepsilon(\mathbf{r} ; t)-$ which is identified with the matrix element $T^{00}(\mathbf{r} ; t)$ of the energy momentum tensor in the local rest frame at space-time $(\mathbf{r}, t)$ - reaches as high as $30 \mathrm{GeV} / \mathrm{fm}^{3}$ in a central $\mathrm{Au}+\mathrm{Au}$ collision at $\sqrt{s}=200 \mathrm{GeV}[1]$.

According to present knowledge, the charmonium production in heavy-ion collisions, i.e. $c \bar{c}$ pairs, occurs exclusively at the initial stage of the reaction in primary nucleon-nucleon collisions. The parametrizations of the total charmonium cross sections $\left(i=\chi_{c}, J / \Psi, \Psi^{\prime}\right)$ from $N N$ collisions as a function of the invariant energy $\sqrt{s}$ used in this work are taken from [2, 3, 4, 5]. We recall that (as in Refs. [4, 5, 6, 17, 8]) the charm degrees of freedom in the HSD approach are treated perturbatively and that initial hard processes (such as $c \bar{c}$ or Drell-Yan production from $N N$ collisions) are 'precalculated' to achieve a scaling of the inclusive cross section with the number of projectile and target nucleons as $A_{P} \times A_{T}$ when integrating over impact parameter. For fixed impact parameter $b$ the $c \bar{c}$ yield then scales with the number of binary hard collisions $N_{b i n}$ ( $c f$. Fig. 8 in Ref. [5]).

In the QGP 'threshold scenario', e.g the geometrical Glauber model of Blaizot et al. [9] as well as the percolation model of Satz [10], the QGP suppression '(i)' sets in rather abruptly as soon as the energy density exceeds a threshold value $\varepsilon_{c}$, which is a free parameter. This version of the standard approach is motivated by the idea that the charmonium dissociation rate is drastically larger in a quark-gluon-plasma (QGP) than in a hadronic medium [10].

On the other hand, the extra suppression of charmonia in the high density phase of nucleus-nucleus collisions at SPS energies [11, 12, 13, 14] has been attributed to inelastic comover scattering ( $c f .[2,3$, 7, 15, 16, 17, 18, 19, 20, 21] and Refs. therein) assuming that the corresponding $J / \Psi$-hadron cross sections are in the order of a few $\mathrm{mb}$ [22, 23, 24, 25]. In these models 'comovers' are viewed not as asymptotic hadronic states in vacuum but rather as hadronic correlators (essentially of vector meson type) that might well survive at energy densities above $1 \mathrm{GeV} / \mathrm{fm}^{3}$. Additionally, alternative absorption mechanisms might play a role, such as gluon scattering on color dipole states as suggested in Refs. [26, 27, 28, 29] or charmonium dissociation in the strong color fields of overlapping strings [6].

The explicit treatment of initial $c \bar{c}$ production by primary nucleon-nucleon collisions and the implementation of the comover model - involving a single matrix element $M_{0}$ fixed by the data at SPS energies - as well as the QGP threshold scenario in HSD were explained in Ref. [1, 15] (see Fig. 1 of Ref. [15] for the relevant cross sections). We recall that the 'threshold scenario' for charmonium dissociation is implemented as follows: whenever the local energy density $\varepsilon(x)$ is above a threshold value $\varepsilon_{j}$ (where the index $j$ stands for $\left.J / \Psi, \chi_{c}, \Psi^{\prime}\right)$, the charmonium is fully dissociated to $c+\bar{c}$. The 


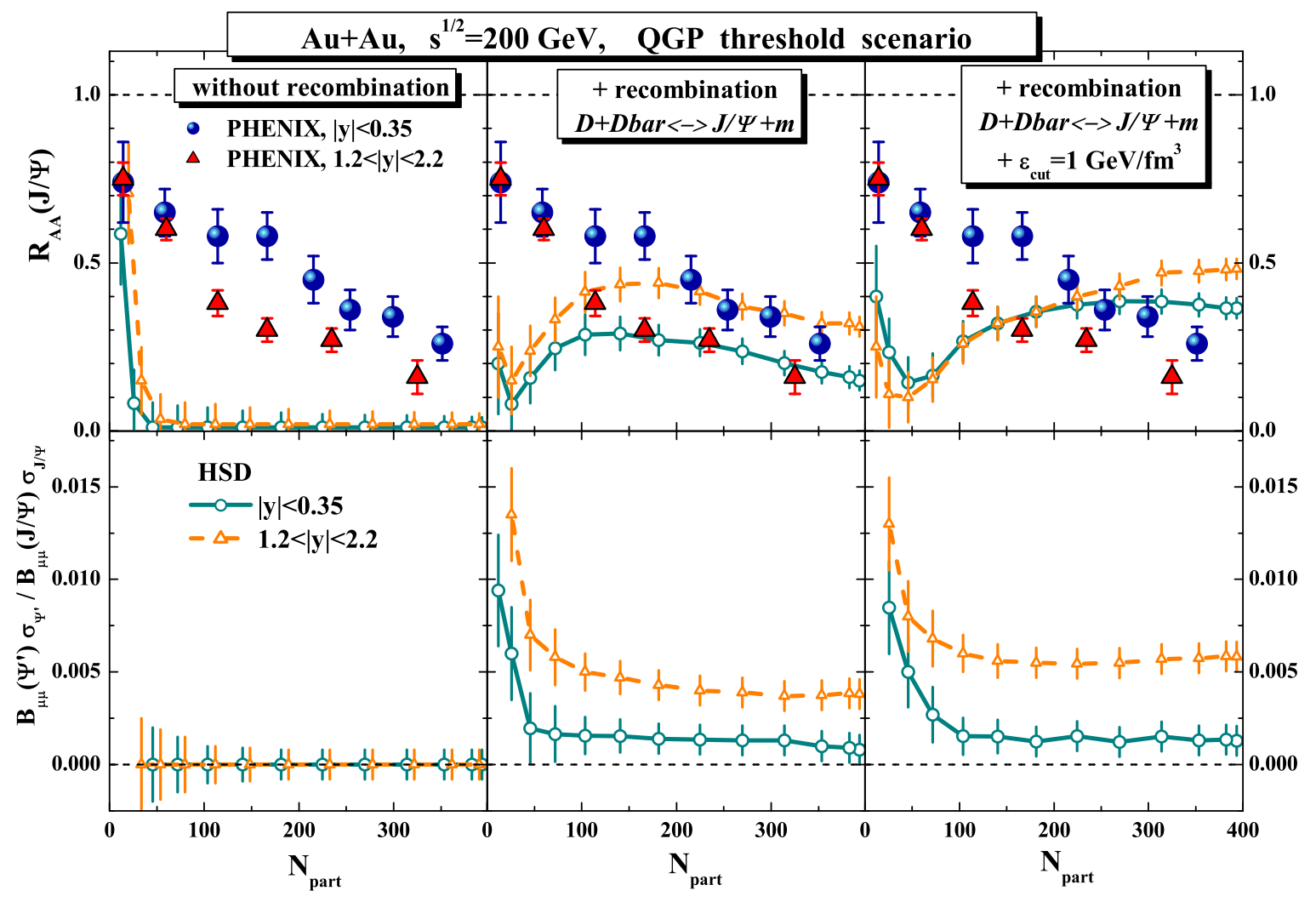

Figure 1. The $J / \Psi$ nuclear modification factor $R_{A A}$ for $A u+A u$ collisions at $\sqrt{s}=200 \mathrm{GeV}$ as a function of the number of participants $N_{\text {part }}$ in comparison to the data from [10] for midrapidity (full circles) and forward rapidity (full triangles). HSD results for the QGP 'threshold melting' scenarios are displayed in terms of the lower (green solid) lines for midrapidity $J / \Psi$ 's $(|y| \leq 0.35)$ and in terms of the upper (orange dashed) lines for forward rapidity $(1.2 \leq y \leq 2.2)$ within different recombination scenarios (see text). The error bars on the theoretical results indicate the statistical uncertainty due to the finite number of events in the HSD calculations. Predictions for the ratio $B_{\mu \mu}\left(\Psi^{\prime}\right) \sigma_{\Psi^{\prime}} / B_{\mu \mu}(J / \Psi) \sigma_{J / \Psi}$ as a function of the number of participants $N_{\text {part }}$ are shown in the lower set of plots. The figure is taken from [1].

default threshold energy densities adopted are $\varepsilon_{1}=16 \mathrm{GeV} / \mathrm{fm}^{3}$ for $J / \Psi, \varepsilon_{2}=2$ $\mathrm{GeV} / \mathrm{fm}^{3}$ for $\chi_{c}$, and $\varepsilon_{3}=2 \mathrm{GeV} / \mathrm{fm}^{3}$ for $\Psi^{\prime}$. Two more scenarios were implemented similarly to the 'comover suppression' and the 'threshold melting' by adding the only additional assumption - that the comoving mesons (including the $D$-mesons) exist only at energy densities below some cut-energy density $\epsilon_{c u t}$, which is a free parameter. We set $\epsilon_{\text {cut }}=1 \mathrm{GeV} / \mathrm{fm}^{3}$, i.e. of the order of critical energy density.

\section{Comparison to data}

In the following, we compare our calculations to the experimental data at the top RHIC energy of $\sqrt{s}=200 \mathrm{GeV}$. We recall that the experimentally measured nuclear 


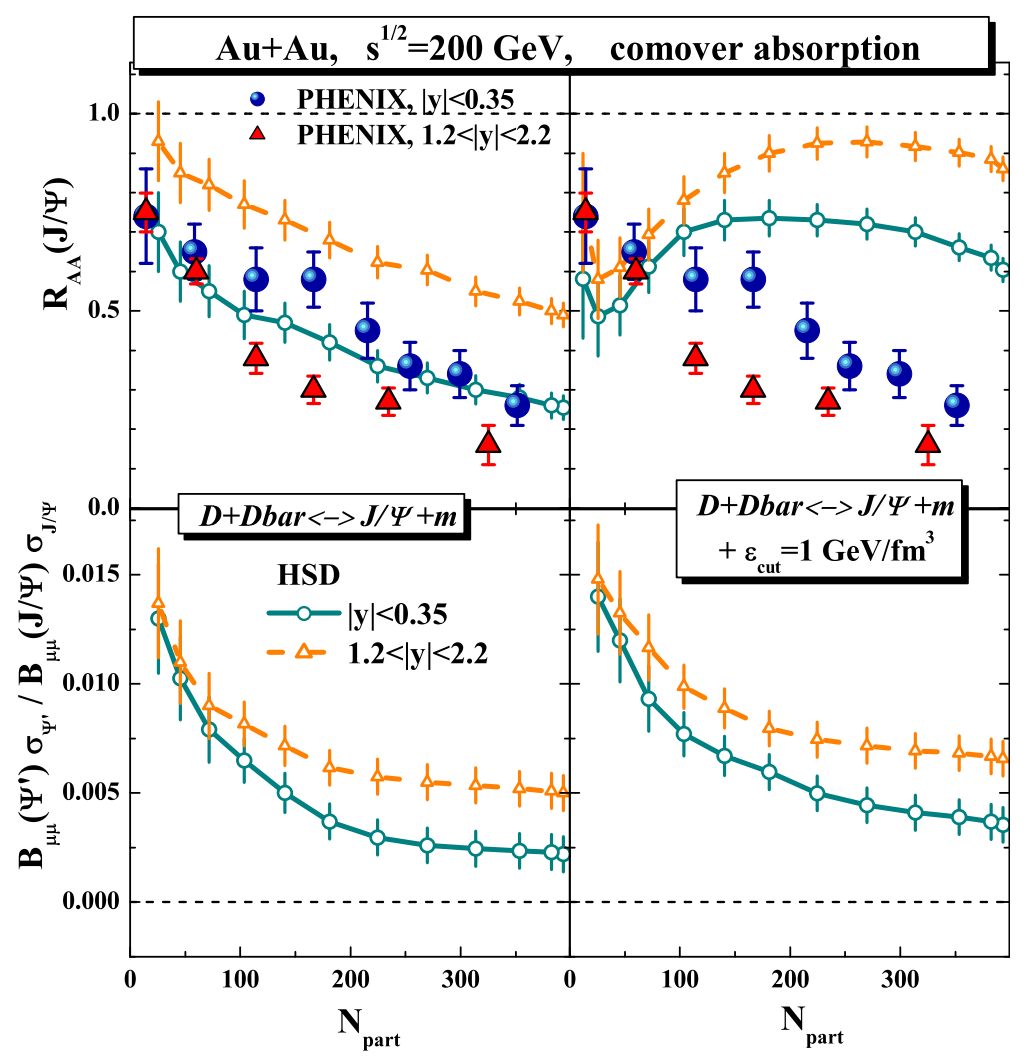

Figure 2. Same as Fig. 4 for the 'comover absorption scenario' including the charmonium reformation channels without cut in the energy density (l.h.s.) and with a cut in the energy density $\epsilon_{\text {cut }}=1 \mathrm{GeV} / \mathrm{fm}^{3}$ (see text for details). The figure is taken from [1.

modification factor $R_{A A}$ is given by

$$
R_{A A}=\frac{d N(J / \Psi)_{A A} / d y}{N_{\text {coll }} \cdot d N(J / \Psi)_{p p} / d y},
$$

where $d N(J / \Psi)_{A A} / d y$ denotes the final yield of $J / \Psi$ in $A A$ collisions, $d N(J / \Psi)_{p p} / d y$ is the yield in elementary $p p$ reactions, $N_{\text {coll }}$ is the number of binary collisions.

Due to very high initial energy density reached (corresponding to $T \gg 2 T_{c}$ ), in the threshold melting scenario all initially created $J / \Psi, \Psi^{\prime}$ and $\chi_{c}$ mesons melt. However, the PHENIX collaboration has found that at least $20 \%$ of $J / \Psi$ do survive at RHIC [30]. Thus, the importance of charmonium recreation is shown again. In HSD, we account for $J / \Psi$ recreation via the $D \bar{D}$ annihilation processes as explained in detail in [1, 15. Note that in our approach, the cross sections of charmonium recreation in $D+\bar{D} \rightarrow J / \Psi+$ meson processes is fixed by detailed balance from the comover absorption cross section $J / \Psi+$ meson $\rightarrow D+\bar{D}$. But even after both these processes are added to the threshold melting mechanism, the centrality dependence of the $R_{A A}(J / \Psi)$ cannot be reproduced in the 'threshold melting' scenario, especially in the peripheral collisions (see Fig. 1). This holds for both possibilities: with (r.h.s. of Fig. 11) and without (center of Fig. 1) the energy density cut $\epsilon_{\text {cut }}$, below which $D$-mesons and comovers exist and 
can participate in $D+\bar{D} \leftrightarrow J / \Psi+$ meson reactions.

Comover absorption scenarios give generally a correct dependence of the yield on the centrality. If an existence of D-mesons at energy densities above $1 \mathrm{GeV} / \mathrm{fm}^{3}$ is assumed, the amplitude of suppression of $J / \Psi$ at mid-rapidity is also well reproduced (see the line for 'comover without $\epsilon_{\text {cut }}$ ' scenario in Fig,2, l.h.s.). Note that this line correspond to the prediction made in the HSD approach in [31]. On the other hand, the rapidity dependence of the comover result is wrong, both with and without $\epsilon_{\text {cut }}$. If hadronic correlators exist only at $\epsilon<\epsilon_{c u t}$, comover absorption is insufficient to reproduce the $J / \Psi$ suppression even at mid-rapidity (see Fig. 2, r.h.s.). But its contribution to the charmonium suppression is, nevertheless, substantial. The difference between the theoretical curves marked 'comover $+\epsilon_{\text {cut }}$ ' and the data shows the maximum possible supression that can be attributed to a deconfined medium.

\section{Summary}

The formation and suppression dynamics of $J / \Psi, \chi_{c}$ and $\Psi^{\prime}$ mesons has been studied within the HSD transport approach for $A u+A u$ reactions at $\sqrt{s}=200 \mathrm{GeV}$. Two currently discussed models, i.e. the 'hadronic comover absorption and reformation' model as well as the 'QGP threshold melting scenario' have been compared to the available experimental data.

We find that both 'comover absorption' and 'threshold melting' fail severely at RHIC energies [1]. The failure of the 'hadronic comover absorption' model goes in line with its underestimation of the collective flow $v_{2}$ of leptons from open charm decay as investigated in Ref. [32]. This suggests that the dynamics of $c, \bar{c}$ quarks at this energy are dominated by strong pre-hadronic interaction of charmonia with the medium in strong QGP (sQGP), which cannot be modeled by 'hadronic' scattering or described appropriately by color screening.

On the other hand, the interaction of $J / \Psi$ with hadrons in the late stages of the collision (when the energy density falls below the critical) gives a sizable contribution to its suppression. This contribution should not be neglected, when comparing possible models for QGP-induced charmonium suppression to the experimental data.

[1] O. Linnyk, E. L. Bratkovskaya, W. Cassing, and H. Stöcker, arXiv:0705.4443[nucl-th].

[2] W. Cassing and E. L. Bratkovskaya, Phys. Rep. 308, 65 (1999).

[3] W. Cassing, E. L. Bratkovskaya, and S. Juchem, Nucl. Phys. A674, 249 (2000).

[4] E. L. Bratkovskaya, W. Cassing, and H. Stöcker, Phys. Rev. C67, 054905 (2003).

[5] W. Cassing, E. L. Bratkovskaya, and A. Sibirtsev, Nucl. Phys. A691, 753 (2001).

[6] J. Geiss, C. Greiner, E. L. Bratkovskaya, W. Cassing, and U. Mosel, Phys. Lett. B447, 31 (1999).

[7] W. Cassing and E. L. Bratkovskaya, Nucl. Phys. A623, 570 (1997).

[8] W. Cassing and C. M. Ko, Phys. Lett. B396, 39 (1997).

[9] J. P. Blaizot and J. Y. Ollitrault, Phys. Rev. Lett. 77, 1703 (1996), hep-ph/9606289.

[10] H. Satz, J. Phys. G32, R25 (2006).

[11] NA50, M. C. Abreu et al., Phys. Lett. B410, 337 (1997).

[12] NA50, M. C. Abreu et al., Phys. Lett. B477, 28 (2000). 
[13] NA50, M. C. Abreu et al., Phys. Lett. B450, 456 (1999).

[14] NA60, A. Foerster et al., J. Phys. G32, S51 (2006), nucl-ex/0609039.

[15] O. Linnyk, E. L. Bratkovskaya, W. Cassing, and H. Stöcker, Nucl. Phys. A786, 183 (2007).

[16] N. Armesto and A. Capella, Phys. Lett. B430, 23 (1998), hep-ph/9705275.

[17] R. Vogt, Phys. Rep. 310, 197 (1999).

[18] C. Gerschel and J. Hüfner, Ann. Rev. Nucl. Part. Sci. 49, 255 (1999).

[19] D. E. Kahana and S. H. Kahana, Prog. Part. Nucl. Phys. 42, 269 (1999).

[20] C. Spieles et al., J. Phys. G25, 2351 (1999), Phys. Rev. C60 (1999) 054901.

[21] L. Gerland, L. Frankfurt, M. Strikman, H. Stöcker, and W. Greiner, Nucl. Phys. A663, 1019 (2000).

[22] K. L. Haglin, Phys. Rev. C61, 031903 (2000).

[23] Z. Lin and C. M. Ko, Phys. Rev. C62, 034903 (2000).

[24] Z. Lin and C. M. Ko, J. Phys. G27, 617 (2001).

[25] A. Sibirtsev, K. Tsushima, and A. W. Thomas, Phys. Rev. C63, 044906 (2001).

[26] B. Zhang, C. M. Ko, B.-A. Li, Z. Lin, and B.-H. Sa, Phys. Rev. C62, 054905 (2000).

[27] L. Grandchamp and R. Rapp, Phys. Lett. B523, 60 (2001), Nucl. Phys. A 709 (2002) 415.

[28] D. Blaschke, Y. Kalinovsky, and V. Yudichev, Lect. Notes Phys. 647, 366 (2004), hep-ph/0410338.

[29] M. Bedjidian et al., hep-ph/0311048.

[30] PHENIX, H. Büsching et al., Nucl. Phys. A774, 103 (2006).

[31] E. L. Bratkovskaya, A. P. Kostyuk, W. Cassing, and H. Stöcker, Phys. Rev. C69, 054903 (2004).

[32] E. L. Bratkovskaya, W. Cassing, H. Stöcker, and N. Xu, Phys. Rev. C71, 044901 (2005). 